\title{
Pion interaction with the trinucleon up to the eta production threshold
}

\author{
S. S. Kamalovt and L. Tiator \\ Institut für Kernphysik, Universität Mainz, 6500 Mainz, Germany \\ C. Bennhold \\ TRIUMF, 4004 Wesbrook Mall, Vancouver, B.C. V6T 2A3, Canada
}

\begin{abstract}
Pion elastic, charge exchange scattering and induced eta production on the trinucleon systems are investigated in a coupled-channels approach in momentum space with Fadeev wave functions. The channel $\pi N \rightarrow \eta N$ is included using an isobar model with S-, P-, and D-wave resonances. While the coherent reactions like ${ }^{3} \mathrm{He}(\pi, \pi)^{3} \mathrm{He}$ can be reasonably well reproduced up to $T_{\pi}=500 \mathrm{MeV}$, large discrepancies appear for the incoherent processes, ${ }^{3} \mathrm{He}\left(\pi^{-}, \pi^{0}\right)^{3} \mathrm{H}$ and ${ }^{3} \mathrm{He}\left(\pi^{-}, \eta\right)^{3} \mathrm{H}$ at backward angles and energies above $\Delta$-resonance. In the forward direction the $(\pi, \eta)$ calculations underestimate the experimental measurements very close to threshold but agreement with the data improves with increasing pion energy. Predictions are made for the asymmetries of the various reactions on polarized ${ }^{3} \mathrm{He}$.
\end{abstract}

PACS numbers: 25.80.Dj, 25.80.Fm, 25.80.Hp

Typeset Using REVTEX 


\section{INTRODUCTION}

For many years there has been a great interest in understanding the pion-nuclear interaction at low and intermediate energies. A recent review of achievements in this field can be found in Ref. [1]. Originally, the phenomenological Kisslinger potential in coordinate space was derived for spin and isospin zero nuclei only as a first step. After that numerous modifications and improvements were suggested to correct the optical potential: real pion absorption, Fermi motion, Lorentz-Lorentz effects, Pauli blocking effects etc.

On the other hand, a microscopic description of the pion-nuclear interaction in momentum space was developed based on the KMT [2] or Watson [3] multiple scattering theories. In the framework of such an approach the nonlocalities of the pion-nuclear interaction, off-shell extrapolations of pion-nucleon scattering amplitudes and exact treatment of Fermi motion have been taken into account [4.5]. In a further step the phenomenological $\rho^{2}$ term has been added [6 8] which is responsible for real pion absorption and second order effects. The momentum space formalism was not only successful in the description of the pion-nuclear interaction in the $\Delta$ resonance region but also at low energies for a large set of nuclei with $\mathrm{A}=4-40$. In Refs. [9, [10] this method has been extended successfully to the description of pionic atoms as well.

At the present time studies of the pion interaction with the trinucleon system have become very attractive. First, this is due to the development of new experimental techniques at TRIUMF, PSI and LAMPF which allow measurements of polarization observables. Secondly, one already has a large set of previous measurements for the differential cross section. On the theoretical side the nuclear structure of the trinucleon system is well known . All these circumstances create ideal conditions to study in detail the pion interaction mechanisms with very light nuclei.

Previous theoretical investigations of the pion interaction with the trinucleon system were mostly based on the multiple scattering theory and, according to a recent analysis by Gibbs and Gibson [11], the KMT version of this approach is preferable. In this frame- 
work Landau [4] and Mach [6] studied the sensitivity of pion elastic scattering and single charge exchange (SCE)on ${ }^{3} \mathrm{He}$ and ${ }^{3} \mathrm{H}$ to the details of the nuclear wave function. While Mach used semiphenomenological wave functions, Landau extracted the four nuclear form factors required for the optical potential from the electromagnetic form factors of ${ }^{3} \mathrm{He}$ and ${ }^{3} \mathrm{H}$. Clearly these two approaches differ since the parametrized form factors contain meson exchange current (MEC) contributions. Nevertheless, both achieve a good description of experimental data up to pion kinetic energy of $T_{\pi}=200 \mathrm{MeV}$.

At higher energies large discrepancies appear between the measurements and theoretical calculations of elastic scattering and SCE at backward angles. For example, in the SCE reaction at $T_{\pi}=300 \mathrm{MeV}$ and at pion angles $\Theta>90^{\circ}$ basically all calculations yield differential cross sections which fall two orders of magnitude below the data. Only calculations in the framework of the Glauber approach 12,13, reached better agreement in this region. However, we concur with Ref. [14]) that in the large-angle region Glauber theory cannot be reliable.

The solution to this problem could involve corrections to the impulse and coherent approximation in form of second order effects. Several attempts to incorporate such corrections for the pion interaction with very light nuclei are discussed in Refs. [14 [17. A full analysis of second order effects including spin and isospin degrees of freedom has been performed by Wakamatsu [14. According to his work these contributions are larger in pion SCE than in elastic scattering and they can enhance the differential cross section at backward angles by a factor of two. This, however, is not sufficient to explain the data.

Another approach was developed by Gibbs and collaborators [11,18]. Here the $s$ - and $p$-wave parts of the first-order potential were calculated using the frozen nucleon approximation, then the obtained potential was averaged over realistic nuclear configurations. In such an approach part of the second-order effects are automatically included via the $N N$ correlations in the nuclear wave function. Refs. [11,18] yield good agreement for pion energies $T_{\pi}=24-180 \mathrm{MeV}$, however, applying this approach to higher energies where higher pion-nucleon partial waves become important encounters computational difficulties. 
The aim of the present work is a systematic investigation of the pion interaction with the trinucleon systems $\left({ }^{3} \mathrm{He}\right.$ and ${ }^{3} \mathrm{H}$ ) in the region of pion kinetic energies of $T_{\pi}=100-600$ $\mathrm{MeV}$, including spin and isospin degrees of freedom on the microscopic level and using only elementary amplitudes extracted from $\pi N$ scattering data and realistic three body wave functions obtained as a solution of the Fadeev equations. Due to the last circumstance we are in a very good position to fix the nuclear structure input in order to shed more light on the reaction mechanism. In the framework of such an approach in our previous studies of pion scattering on unpolarized and polarized ${ }^{3}$ He-targets [19,20] we have reached a good description of older and recent measurements at low pion energies. This has motivated us to extend our momentum space approach for pion energies up to the eta production region. This is the kinematical region $T_{\pi}<600 \mathrm{MeV}$ and momentum transfer $Q<6 \mathrm{fm}^{-1}$, where Fadeev wave functions give an excellent description of the electromagnetic form factors in the $\mathrm{A}=3$ system. This gives us confidence to study the reaction mechanism. In this kinematical region we will check our model by comparison with all available experimental data and present predictions for forthcoming experiments at LAMPF, PSI and TRIUMF.

The main aspects of our formalism based on the KMT multiple scattering approach and coupled-channels method are given in section 2 . In section 3 we describe the nuclear models: 1) a simple S-shell model which is very useful for a qualitative (and in some kinematical regions even quantitative) description of the main features of the pion interaction with the trinucleon [20]; 2) a phenomenological model employing nuclear form factors extracted from electron scattering data which was often used in previous calculations; 3) a three-body model with realistic wave functions obtained from solutions of the Fadeev equations [21]. The aim of this comparison with different nuclear models is to estimate the upper limit of uncertainty which was present in earlier investigations of pion interaction with ${ }^{3} \mathrm{He}$.

In section 4, our results for elastic scattering and single charge exchange on unpolarized and polarized targets are discussed. Furthermore, we briefly consider the "superratio" which has been used [11] to find charge symmetry breaking in strong interactions.

We will complete our analysis with the investigation of eta production by pions. This 
is a relatively new field of theoretical [22 24] and experimental [25] study in pionic physics which promises to be very useful to obtain a global picture of the meson-nuclear interaction. On the other hand, these studies could give very important information on the excitation of nucleon resonances in nuclei (like the $S_{11}(1535)$ and the $D_{13}(1525)$ ) that could be applied to other nuclear reactions with eta mesons (i.e., eta production in heavy-ion collisions and eta photoproduction )

Our results and conclusions are summarized in section 5 and we will give the main expressions for the pion-nucleon and eta-nucleon interactions in the Appendix.

\section{FORMALISM}

\section{A. General expressions}

The general formalism for the description of the pion-nuclear scattering is based on a momentum space coupled-channels method which was developed in detail in Ref. [26]. Here we merely summarize its main features.

In the framework of the KMT version of multiple scattering theory, the scattering amplitude is constructed by solving the Lippmann-Schvinger integral equation with relativistic kinematics:

$$
\mathrm{F}_{\pi^{\prime} \pi}\left(\vec{q}^{\prime}, \vec{q}\right)=\mathrm{V}_{\pi^{\prime} \pi}\left(\vec{q}^{\prime}, \vec{q}\right)-\frac{a}{(2 \pi)^{2}} \sum_{\pi^{\prime \prime}} \int \frac{d \vec{q}^{\prime \prime}}{\mathcal{M}\left(q^{\prime \prime}\right)} \frac{\mathrm{V}_{\pi^{\prime} \pi^{\prime \prime}}\left(\vec{q}^{\prime}, \vec{q}^{\prime \prime}\right) \mathrm{F}_{\pi^{\prime \prime} \pi}\left(\vec{q}^{\prime \prime}, \vec{q}\right)}{E(q)-E\left(q^{\prime \prime}\right)+i \epsilon}
$$

where $\vec{q}$ is the pion momentum, and $E(q)=E_{\pi}(q)+E_{A}(q)$ is the total pion-nuclear energy. The pion-nuclear reduced mass is given by $\mathcal{M}(q)=E_{\pi}(q) E_{A}(q) / E(q)$ and the coefficient $a=(A-1) / A$ is important to avoid double counting.

The momentum space potential of pion-nuclear interaction

$$
\mathrm{V}_{\pi^{\prime} \pi}\left(\vec{q}^{\prime}, \vec{q}\right)=\mathrm{V}_{\text {Coul. }}\left(\vec{q}^{\prime}-\vec{q} ; R\right)+\mathrm{V}_{\pi^{\prime} \pi}^{(1)}\left(\vec{q}^{\prime}, \vec{q}\right)
$$

contains the Coulomb potential in the momentum space, cut at point $\mathrm{R}$, and a potential of strong pion-nuclear interaction $\mathrm{V}_{\pi^{\prime} \pi}^{(1)}$ which is related to the free $\pi N$ scattering t-matrix: 


$$
\mathrm{V}_{\pi^{\prime} \pi}^{(1)}\left(\vec{q}^{\prime}, \vec{q}\right)=-\frac{\sqrt{\mathcal{M}\left(q^{\prime}\right) \mathcal{M}(q)}}{2 \pi}<\pi^{\prime}\left(\vec{q}^{\prime}\right), f\left|\sum_{j=1}^{A} \hat{\mathrm{t}}_{\pi N}(j)\right| i, \pi(\vec{q})>,
$$

where $\mid i>$ and $\mid f>$ denote the nuclear initial and final states, respectively, and $j$ refers to the individual target nucleons.

The pion-nucleon scattering t-matrix is defined in the following way

$$
\hat{\mathrm{t}}_{\pi N}=-\frac{2 \pi}{\sqrt{\mu\left(q^{\prime}, p^{\prime}\right) \mu(q, p)}}\left[A_{00}+A_{01} \vec{t} \cdot \vec{\tau}+i \vec{\sigma} \cdot\left[\hat{\vec{q}}_{i} \times \hat{\vec{q}}_{f}\right]\left(A_{10}+A_{11} \vec{t} \cdot \vec{\tau}\right)\right],
$$

where $\mu(q, p)=E_{\pi}(q) E_{N}(p) / \omega$ is the pion-nucleon reduced mass, $\vec{p}$ and $\vec{p}^{\prime}=\vec{p}+\vec{q}-\vec{q}^{\prime}$ are the nucleon momenta in the initial and final states, respectively, and $\hat{\vec{q}}_{i}$ and $\hat{\vec{q}}_{f}$ are the unit vectors for the initial and final pion momentum in the $\pi N$ c.m. system. The vectors $\vec{\sigma}, \vec{\tau}$ and $\vec{t}$ are the usual spin and isospin operators of target nucleon and pion, respectively.

The scalar functions $A_{S T}\left(\omega, \cos \Theta^{*}\right)(S=0,1$ and $T=0,1)$, which depend on the total pion-nucleon energy $\omega$ and the pion angle $\Theta^{*}$ in the $\pi N$ c.m. system, are the usual combinations of partial $\pi N$ scattering amplitudes $f_{l_{\pi}}^{( \pm)}(\omega)$ and Legendre polynomials $P_{l_{\pi}}\left(\cos \Theta^{*}\right)$ where $l_{\pi}$ is the pion-nucleon angular momentum and $( \pm)$ corresponds to the total spin $j_{\pi}=l_{\pi} \pm \frac{1}{2}$ of the $\pi N$ system. These amplitudes are constructed from the pion-nucleon phase-shift parametrization of ref. [27] at $m_{\pi}+M_{N}<\omega<1.3 \mathrm{GeV}$ and the CERNTHparametrization [28] at $1.3 \mathrm{GeV}<\omega<2.2 \mathrm{GeV}$.

The discussion of all problems connected with the partial wave analysis of different groups is beyond this paper. A review of this subject can be found in the last compilation of [29]. Note only that in the kinematical region considered here $(\omega<1.6 \mathrm{GeV})$, the difference between the results of partial wave analysis of different groups is small (much smaller than the accuracy of the nuclear data). For example, a comparison of CERNTH and VPI [30] analyses show no difference in the P33 wave up to $w=1.6 \mathrm{GeV}$. Also P11 and D13 phase shifts are very similar in this region. Only for $S 11$ the difference between results of these two groups becomes sizable at $w>1.5 \mathrm{GeV}$ [22], i.e. in the region were the eta channel is open. Note that recently a new $\pi N$ scattering partial analysis was published by the VPI group 31 which gives a new determination of $S 11$ resonance parameters consistent with 
results of other groups.

The off-shell extrapolation of $\pi N$ parcial amplitudes was obtained using the separable form

$$
f_{l_{\pi}}^{( \pm)}(\text {off }- \text { shell })=f_{l_{\pi}}^{( \pm)}(\omega) \frac{v_{l_{\pi}}^{( \pm)}\left(q^{\prime \prime}\right) v_{l_{\pi}}^{( \pm)}\left(q^{\prime}\right)}{\left[v_{l_{\pi}}^{( \pm)}(q)\right]^{2}}
$$

with $\pi N$ form factors

$$
v_{l_{\pi}}^{( \pm)}(q)=\frac{q^{l_{\pi}}}{\left[1+\left(r_{0} q\right)^{2}\right]^{2}}
$$

Here we employ the value $r_{0}=0.47 \mathrm{fm}$ consistent with the analysis of the separable $\pi N$ potential in Ref. [32].

To connect the total pion-nucleon energy $\omega$, at which these partial amplitudes are calculated, with the total pion-nuclear energy $E$ we will use the results of a relativistic generalization for the three-body model [6, 26]:

$$
\begin{aligned}
\omega= & E+m_{\pi}+M_{N}-\left\{\left(m_{\pi}+M_{N}\right)^{2}+\left[\frac{A-1}{2 A}\left(\vec{q}^{\prime}+\vec{q}\right)^{2}\right]^{2}\right\}^{\frac{1}{2}}- \\
& -\left\{(A-1)^{2} M_{N}^{2}+\left[\frac{A-1}{2 A}\left(\vec{q}^{\prime}+\vec{q}\right)^{2}\right]^{2}\right\}^{\frac{1}{2}} .
\end{aligned}
$$

Such an approach allowed us to improve the impulse approximation in accordance with the results of Ref. [33].

\section{B. Partial wave decomposition and the amplitudes $\mathcal{F}$ and $\mathcal{G}$}

In our numerical applications we express the scattering amplitude in terms of partial amplitudes using the representations of total isospin $I$ and projection $\xi$

$$
\mathrm{F}_{\pi^{\prime} \pi}\left(\vec{q}^{\prime}, \vec{q}\right)=\sum_{I, \xi}(2 I+1)\left(\begin{array}{ccc}
1 & \frac{1}{2} & I \\
\pi & \nu_{i} & -\xi
\end{array}\right)\left(\begin{array}{ccc}
1 & \frac{1}{2} & I \\
\pi^{\prime} & \nu_{f} & -\xi
\end{array}\right) \mathrm{F}_{I}\left(\vec{q}^{\prime}, \vec{q}\right)
$$

and of total angular momentum $j$ with projection $m$

$$
\mathrm{F}_{I}\left(\vec{q}^{\prime}, \vec{q}\right)=4 \pi \sum(2 j+1) Y_{M_{\pi}^{\prime}}^{L_{\pi}}\left(\hat{\overrightarrow{q^{\prime}}}\right) F_{L_{\pi}}^{j, I}\left(q^{\prime}, q\right) Y_{M_{\pi}}^{* L_{\pi}}(\hat{\vec{q}})\left(\begin{array}{ccc}
L_{\pi} & \frac{1}{2} & j \\
M_{\pi}^{\prime} & M_{f} & -m
\end{array}\right)\left(\begin{array}{ccc}
L_{\pi} & \frac{1}{2} & j \\
M_{\pi} & M_{i} & -m
\end{array}\right),
$$


where $\left(\begin{array}{l}\cdots \\ \ldots\end{array}\right)$ stand for the $3 j$ symbol, $\nu_{i}, \nu_{f}$ are the isospin projectors of the initial and final nucleus, $Y_{M_{\pi}}^{L_{\pi}}(\hat{\vec{q}})$ are spherical harmonics for the pion waves and $L_{\pi}$ is the pion angular momentum. Note that in the case of isoelastic pion scattering on the $A=3$ system the pion orbital angular momentum in the initial and final states are equal due to parity conservation. For the potential $\mathrm{V}_{\pi^{\prime} \pi}$ we perform an expansion identical to eqs. (8,9).

Substituting the above expansions of $\mathrm{V}_{\pi^{\prime} \pi}$ and $\mathrm{F}_{\pi^{\prime} \pi}$ into eq.(1) we obtain the following integral equation for the partial wave amplitudes

$$
F_{L_{\pi}}^{j, I}\left(q^{\prime}, q\right)=V_{L_{\pi}}^{j, I}\left(q^{\prime}, q\right)-\frac{a}{\pi} \int \frac{q^{\prime \prime 2}}{\mathcal{M}\left(q^{\prime \prime}\right)} \frac{V_{L_{\pi}}^{j, I}\left(q^{\prime}, q^{\prime \prime}\right) F_{L_{\pi}}^{j, I}\left(q^{\prime \prime}, q\right)}{E(q)-E\left(q^{\prime \prime}\right)+i \epsilon} d q^{\prime \prime}
$$

This equation is solved using the matrix inversion method. To obtain the final values for the partial amplitudes we also take into account the Coulomb interaction applying the matching procedure developed by Vincent and Phatak 34.

Now let us consider some isospin symmetry properties that follow from eq.(8) when the Coulomb force is turned off. Overall, this is a good approximation except for the small angle region. In this isospin symmetric case for the $A=3$ system we have the following relations: for the $\pi^{ \pm}$elastic scattering amplitudes

$$
\begin{gathered}
<\pi^{+},{ }^{3} \mathrm{He}|\mathrm{F}|{ }^{3} \mathrm{He}, \pi^{+}>=<\pi^{-},{ }^{3} \mathrm{H}|\mathrm{F}|{ }^{3} \mathrm{H}, \pi^{-}>=\mathrm{F}_{\frac{3}{2}} \\
<\pi^{-},{ }^{3} \mathrm{He}|\mathrm{F}|{ }^{3} \mathrm{He}, \pi^{-}>=<\pi^{+},{ }^{3} \mathrm{H}|\mathrm{F}|{ }^{3} \mathrm{H}, \pi^{+}>=\frac{1}{3}\left(\mathrm{~F}_{\frac{3}{2}}+2 \mathrm{~F}_{\frac{1}{2}}\right)
\end{gathered}
$$

and for the single charge exchange reaction

$$
<\pi^{0},{ }^{3} \mathrm{H}|\mathrm{F}|{ }^{3} \mathrm{He}, \pi^{-}>=<\pi^{0},{ }^{3} \mathrm{He}|\mathrm{F}|{ }^{3} \mathrm{H}, \pi^{+}>=\frac{\sqrt{2}}{3}\left(\mathrm{~F}_{\frac{3}{2}}-\mathrm{F}_{\frac{1}{2}}\right)
$$

From relations (11) it follows that the pion interaction with the $\mathrm{A}=3$ systems is described by the independent amplitudes $F_{\frac{1}{2}}$ and $F_{\frac{3}{2}}$ which correspond to scattering in the channels with total pion-nuclear isospins $I=\frac{1}{2}$ and $\frac{3}{2}$, respectively. In general, these amplitudes are complex. Moreover, the inclusion of spin degrees of freedom leads to their additional decomposition into non spin-flip and spin-flip parts. Therefore, studying only differential 
cross sections for elastic scattering and the SCE reactions is not enough to obtain full information about these amplitudes.

Using the spin structure of the free $\pi N$ scattering amplitude (see eq.(4)) the isotopic $\mathrm{F}_{I}$ amplitude can be represented as

$$
\mathrm{F}_{I}(q, \cos \Theta)=\mathcal{F}_{I}(q, \cos \Theta)+i \mathcal{G}_{I}(q, \cos \Theta) \sin \Theta \vec{\sigma} \cdot \hat{n}
$$

Here we assumed (in accordance with the Madison convention) that the incident pion momentum $\vec{q}$ is along the positive z-axis and the vector $\hat{n}=\left(\vec{q} \times \vec{q}^{\prime}\right) /\left|\vec{q} \times \vec{q}^{\prime}\right|$ is along the positive $\mathrm{y}$-axis in the right-handed coordinate system.

In the plane wave impulse approximation the amplitudes $\mathcal{F}_{I}$ and $\mathcal{G}_{I}$ are directly connected with the non spin-flip $\left(A_{00}, A_{01}\right)$ and spin-flip $\left(A_{10}, A_{11}\right)$ parts of the elementary amplitude of eq.(4) (see also next section). But taking into account the contributions of pion rescattering by solving the integral equation (10) leads to a more complicated situation. In this case both non spin-flip and spin-flip parts of the elementary amplitude give contributions to $\mathcal{F}_{I}$ and $\mathcal{G}_{I}$.

Expressions for the total amplitudes $\mathcal{F}$ and $\mathcal{G}$ can be obtained by summing the partial amplitudes in eqs.(9) and (10). They are the same as in $\pi N$ scattering, i.e.

$$
\begin{gathered}
\mathcal{F}=\mathcal{F}_{\text {Coul. }}+\sum_{L_{\pi}} \exp \left(2 i \delta_{L_{\pi}}^{C}\right)\left[\left(L_{\pi}+1\right) F_{L_{\pi}}^{(+)}(q)+L_{\pi} F_{L_{\pi}}^{(-)}(q)\right] P_{L_{\pi}}(\cos \Theta) \\
\mathcal{G}=\sum_{L_{\pi}} \exp \left(2 i \delta_{L_{\pi}}^{C}\right)\left[F_{L_{\pi}}^{(+)}(q)-F_{L_{\pi}}^{(-)}(q)\right] P_{L_{\pi}}^{\prime}(\cos \Theta)
\end{gathered}
$$

where $\delta_{L_{\pi}}^{C}$ is the point Coulomb phase shift, $P_{L}$ and $P_{L}^{\prime}$ are the Legendre polynomial and its derivative, respectively, and $( \pm)$ corresponds to the total spin $j=L_{\pi} \pm \frac{1}{2}$ of the $\pi A$ system. For the point Coulomb phase shifts $\delta_{L_{\pi}}^{C}$ and the amplitude $\mathcal{F}_{\text {Coul. we }}$ used standard expressions given in Ref. 34

The differential cross section gives information on the incoherent sum of $\mathcal{F}$ and $\mathcal{G}$, namely

$$
\frac{d \sigma}{d \Omega}=|\mathcal{F}|^{2}+|\mathcal{G}|^{2} \sin ^{2} \Theta
$$


On the other hand, polarization observables offer the possibility of learning more about these two complex angle-dependent functions. For example, the asymmetry observable depends on their interference

$$
A_{y}=\frac{2 \operatorname{Im}\left(\mathcal{F} \mathcal{G}^{*}\right) \sin \Theta}{|\mathcal{F}|^{2}+|\mathcal{G}|^{2} \sin ^{2} \Theta}
$$

The real part could in principle be measured in a double polarization experiment by detecting the recoil polarization of the final nucleus after pion scattering from a polarized target.

The full analysis of differential cross sections and polarization observables for all the reactions listed in eq.(11) can give us complete information about the spin and isospin parts of the pion interaction with the $A=3$ system. Thus we have the opportunity to test their symmetry properties and to obtain new information about the nature of the pion-nuclear interaction.

\section{NUCLEAR MODELS}

\section{A. The three-body model}

Now we turn to the potential $\mathrm{V}_{\pi^{\prime} \pi}$ defined in eqs.(3,4). For the trinucleon system the nuclear wave functions which enter this expression have to be given by Fadeev calculations with realistic nucleon-nucleon potentials. In our calculations we use a wave function which has been obtained in Ref. [21] with the Reid soft-core potential. This wave function describes both static and dynamical properties of the $\mathrm{A}=3$ system at momentum transfers $0<Q<$

$5-6 \mathrm{fm}^{-1}$, provided one includes meson exchange currents in electromagnetic observables.

Using this three-body wave function we can rewrite the expression (3) for the potential $\mathrm{V}_{\pi^{\prime} \pi}$ in the following way 35 ,

$$
\mathrm{V}_{\pi^{\prime} \pi}\left(\vec{q}^{\prime}, \vec{q}\right)=-3 \frac{\sqrt{\mathcal{M}\left(q^{\prime}\right) \mathcal{M}(q)}}{2 \pi} \int d \vec{p} d \vec{P} \Psi_{f}^{*}\left(\vec{P}, \vec{p}^{\prime}\right) \hat{t}\left(\omega, \overrightarrow{q^{\prime}}, \vec{q}, \vec{p}_{1}\right) \Psi_{i}(\vec{P}, \vec{p}),
$$

where the arguments of the nuclear wave functions are the Lovelace coordinates 


$$
\vec{p}=-\frac{\sqrt{3}}{2} \vec{p}_{1}, \quad \vec{p}^{\prime}=\vec{p}-\frac{\vec{Q}}{\sqrt{3}}, \quad \vec{P}=\frac{1}{2}\left(\vec{p}_{2}-\vec{p}_{3}\right)
$$

with the momentum transfer $\vec{Q}=\vec{q}-\vec{q}^{\prime}$.

The nuclear wave functions $\Psi(\vec{P}, \vec{p})$ are expanded in orbital momentum, spin, and isospin space as

$$
\Psi(\vec{P}, \vec{p})=\sum_{\alpha} \phi_{\alpha}(P, p)\left|(\tilde{L} l) \mathcal{L},\left(\tilde{S} \frac{1}{2}\right) \mathcal{S}, \frac{1}{2} M>\right|\left(\tilde{T} \frac{1}{2}\right) \frac{1}{2} \nu>
$$

where $\phi_{\alpha}(P, p)$ are numerical solutions of the Fadeev equations. To shorten the notation we introduced $\alpha=\{\tilde{L} l \mathcal{L} \tilde{S} \mathcal{S} \tilde{T}\}$, where $\tilde{L}, \tilde{S}$ and $\tilde{T}$ are the total angular momentum, spin, and isospin of the pair $(2,3)(\tilde{L}$ is associated with momentum $\vec{P})$, and $l$ and $\frac{1}{2}$ have an analogius meaning for the particle $(1)$ ( $l$ is associated with momentum $\vec{p})$.

Nucleonic Fermi motion is treated as in Refs. [35,36] where it was found that the substitution

$$
\vec{p}_{1} \rightarrow \vec{p}_{\text {eff. }}=-\frac{\vec{q}}{A}-\frac{A-1}{2 A}\left(\vec{q}-\vec{q}^{\prime}\right)
$$

(factorization approximation) provides numerical results very close to the exact ones. This approximation allows us to express the isospin dependent potential $\mathrm{V}_{I}\left(\vec{q}^{\prime}, \vec{q}\right)$ in the form

$$
\mathrm{V}_{I}=4 \pi W_{A}(-1)^{\frac{1}{2}-M_{f}} \sum_{S T L J} i^{L+S} C_{I}^{T} A_{S T} M_{S L}^{J T}(Q)\left[K^{S} \times Y^{* L}(\hat{\vec{Q}})\right]_{M_{J}}^{J}\left(\begin{array}{ccc}
\frac{1}{2} & J & \frac{1}{2} \\
-M_{f} & M_{J} & M_{i}
\end{array}\right)
$$

where $A_{S T}\left(\omega, \cos \Theta^{*}\right)$ are the $\pi N$ scattering amplitudes defined in eq.(4), $K^{0}=1$ and $K^{1}=\left[\hat{\vec{q}}_{i} \times \hat{\vec{q}}_{f}\right]$, and $C_{I}^{T}$ and $W_{A}$ stand for the

isospin

$$
C_{1 / 2}^{T}=(-1)^{T} \sqrt{\frac{T+1}{T+2}}, \quad C_{3 / 2}^{T}=\sqrt{\frac{1}{2(2 T+1)}}
$$

and kinematical

$$
W_{A}\left(\vec{q}^{\prime}, \vec{q}\right)=\sqrt{\frac{\mathcal{M}\left(q^{\prime}\right) \mathcal{M}(q)}{\mu\left(\vec{q}^{\prime}, \vec{p}_{1}^{\prime}\right) \mu\left(\vec{q}, \vec{p}_{1}\right)}}
$$

factors. Using Fadeev wave functions from eq.(18) we obtained the following expression for the nuclear form factor $M_{S L}^{J T}(Q)$, 


$$
\begin{aligned}
& M_{S L}^{J T}(Q)=\frac{3}{\sqrt{4 \pi}} i^{-L} \sum_{\alpha^{\prime}, \alpha} O_{S L}^{J T}\left(\alpha^{\prime}, \alpha\right) \int d \vec{p}\left[Y^{l^{\prime}}\left(\Omega_{\vec{p}^{\prime}}\right) \times Y^{l}\left(\Omega_{\vec{p}}\right)\right]_{0}^{L} \\
& \times \int P^{2} d P \phi_{\alpha^{\prime}}\left(P, p^{\prime}\right) \phi_{\alpha}(P, p), \\
& O_{S L}^{J T}\left(\alpha^{\prime}, \alpha\right)=8 \hat{\mathcal{L}} \hat{\mathcal{L}}^{\prime} \hat{\mathcal{S}} \hat{\mathcal{S}}^{\prime} \hat{\tilde{S}} \hat{L} \hat{T} \delta_{\tilde{L} \tilde{L}^{\prime}} \delta_{\tilde{S} \tilde{S}^{\prime}} \delta_{\tilde{T} \tilde{T}^{\prime}}(-1)^{l+l^{\prime}+\tilde{L}-\mathcal{L}+\mathcal{S}+\tilde{S}+\tilde{T}+S+T+3 / 2} \\
& \times\left\{\begin{array}{ccc}
\frac{1}{2} & \frac{1}{2} & S \\
\mathcal{S}^{\prime} & \mathcal{S} & \tilde{S}
\end{array}\right\}\left\{\begin{array}{ccc}
\mathcal{L} & \mathcal{L}^{\prime} & L \\
l^{\prime} & l & \tilde{L}
\end{array}\right\}\left\{\begin{array}{ccc}
\frac{1}{2} & \tilde{T} & \frac{1}{2} \\
\frac{1}{2} & T & \frac{1}{2}
\end{array}\right\}\left\{\begin{array}{ccc}
\mathcal{L} & \mathcal{S} & \frac{1}{2} \\
\mathcal{L}^{\prime} & \mathcal{S}^{\prime} & \frac{1}{2} \\
L & S & J
\end{array}\right\}
\end{aligned}
$$

\section{B. The phenomenological model}

In eq.(23) parity and angular momentum selection rules determine which nuclear form factors can contribute. In analogy with electromagnetic form factors we refer to them as $S=0, \quad L=0, \quad J=0, \quad T=0$ or 1 -isoscalar or isovector C0 form factor $S=1, \quad L=0,2 \quad J=1, \quad T=0$ or 1 -isoscalar or isovector M1 form factor

In fact, assuming that mesonic exchange currents (MEC) contributions are small in elastic electron scattering these form factors had been extracted directly from the electromagnetic form factors of ${ }^{3} \mathrm{He}$ and ${ }^{3} \mathrm{H}$ [, 37]. It was believed that this assumption is more reliable for the matter distribution described by $\mathrm{C} 0$ form factors. However, recent theoretical investigations of the charge distributions of trinucleon systems show that MEC contributions in $\mathrm{C} 0$ transitions at transfer momentum $Q^{2}>5 \mathrm{fm}^{-2}$ are important too [38]. Therefore, the connection between matter distribution and experimental charge form factors is not straightforward as it was suggested in many previous calculations.

In this paper we try to estimate the upper limit of uncertainty, which was present in early investigations by comparison of results obtained in phenomenological models with 
microscopical calculations. In our phenomenological model we express the $\mathrm{C} 0$ form factors by the charge form factors of ${ }^{3} \mathrm{He}\left(\mathrm{F}_{c h}^{3 \mathrm{He}}\right)\left[39\right.$ and ${ }^{3} \mathrm{H}\left(\mathrm{F}_{c h}^{3} \mathrm{H}\right)[40$ ]:

$$
M_{00}^{0 T}(Q)=\frac{1}{\sqrt{\pi}} \hat{T}\left[2 \mathrm{~F}_{c h}^{3 \mathrm{He}}(Q)+(-1)^{T} \mathrm{~F}_{c h}^{3 \mathrm{H}}(Q)\right] / \mathrm{f}_{c h}^{p}(Q),
$$

where $\mathrm{f}_{c h}^{p}(Q)$ is the proton charge form factor.

For the M1 form factors we will keep the microscopical description via the Fadeev wave function, because here the phenomenological approach is clearly not applicable. First, there are large MEC contributions in the M1 electron scattering form factor, and secondly, it is not possible to unambiguously separate the contributions from spin and convection currents. Therefore, we will use the Fadeev wave functions described in the previous subsection for the $\mathrm{J}=1$ transition densities.

\section{The simple S-shell model}

Finally, we employ a very simple model where the nucleons are in the $0 s_{1 / 2}$ shell of a harmonic oscillator potential and, furthermore, pion rescattering and Coulomb contributions

are neglected, e.g. $\mathrm{F}_{\pi^{\prime} \pi}=\mathrm{V}_{\pi^{\prime} \pi}^{(1)}$ (plane wave approximation). The last assumption allows expressing $\mathcal{F}_{I}$ and $\mathcal{G}_{I}$, introduced in the previous section, directly via the non spin-flip ( $\left.A_{00}, A_{01}\right)$ and spin flip $\left(A_{10}, A_{11}\right)$ components of the $\pi N$ elementary amplitude of eq.(4).

Evaluating eq.(20) in the frame with the initial pion momentum $\vec{q}$ along the z-axis and the vector $\left[\vec{q} \times \vec{q}^{\prime}\right]$ along the y-axis (as in eqs.(12-15)) the plane wave expressions for $\mathcal{F}_{I}$ and $\mathcal{G}_{I}$ can be written as

$$
\begin{gathered}
\mathcal{F}_{I}=\sqrt{2 \pi} W_{A} \sum_{T} C_{I}^{T} A_{0 T}\left(\omega, \cos \Theta^{*}\right) M_{00}^{0 T}(Q) \\
\mathcal{G}_{I}=\sqrt{\frac{2 \pi}{3}} W_{A} W_{B} \sum_{T} C_{I}^{T} A_{1 T}\left(\omega, \cos \Theta^{*}\right)\left[M_{10}^{1 T}(Q)-\frac{1}{\sqrt{2}} M_{12}^{1 T}(Q)\right],
\end{gathered}
$$

where $W_{B}$ is an additional kinematical factor arising due to the Lorentz transformation of the vector $\left[\hat{\vec{q}}_{i} \times \hat{\vec{q}}_{f}\right]$ in the elementary amplitude from the $\pi N$ c.m. to the $\pi A$ c.m. system. 
Note that the expressions (25) are valid not only for ${ }^{3} \mathrm{He}$ or ${ }^{3} \mathrm{H}$ but for all nuclei with spin and isospin one half $\left({ }^{13} \mathrm{C},{ }^{15} \mathrm{~N}, \ldots\right)$

In the framework of the S-shell model the nuclear form factors $M_{S L}^{J T}(Q)$ are simply given by

$$
M_{S L}^{J T}(Q)=\frac{1}{\sqrt{\pi}} \hat{T} \hat{S} R_{S}(Q) \delta_{L, 0} \Psi_{J T}
$$

where $R_{S}(Q)=\exp \left(-b^{2} Q^{2} / 6\right)$ with $b=1.65 \mathrm{fm}$ as the point radius of ${ }^{3} \mathrm{He}$, and the coefficients are $\Psi_{00}=3$ and $\Psi_{01}=\Psi_{10}=-\Psi_{11}=1$. In this case we can express $\mathcal{F}$ and $\mathcal{G}$ for the reactions (11) via the analogous amplitudes $f$ and $g$ for $\pi N$ scattering:

for elastic $\pi^{ \pm} \_{ }^{3} \mathrm{He}$ or $\pi^{\mp} \_{ }^{3} \mathrm{H}$ scattering

$$
\begin{gathered}
\mathcal{F}=\left(2 f_{\pi^{ \pm} \mathrm{p}}+f_{\pi^{ \pm} \mathrm{n}}\right) R_{S}(Q) W_{A}=\left(3 A_{00} \pm A_{01}\right) R_{S}(Q) W_{A} \\
\mathcal{G}=g_{\pi^{ \pm} \mathrm{n}} R_{S}(Q) W_{A} W_{B}=\left(A_{10} \mp A_{11}\right) R_{S}(Q) W_{A} W_{B}
\end{gathered}
$$

for the ${ }^{3} \mathrm{He}\left(\pi^{-}, \pi^{0}\right)^{3} \mathrm{H}$ or ${ }^{3} \mathrm{H}\left(\pi^{+}, \pi^{0}\right)^{3} \mathrm{He}$ reactions

$$
\begin{gathered}
\mathcal{F}=\frac{1}{\sqrt{2}}\left(f_{\pi^{+} \mathrm{p}}-f_{\pi^{+} \mathrm{n}}\right) R_{S}(Q) W_{A}=\sqrt{2} A_{01} R_{S}(Q) W_{A} \\
\mathcal{G}=-\frac{1}{\sqrt{2}}\left(g_{\pi^{+} \mathrm{p}}-g_{\pi^{+} \mathrm{n}}\right) R_{S}(Q) W_{A} W_{B}=-\sqrt{2} A_{11} R_{S}(Q) W_{A} W_{B} .
\end{gathered}
$$

The above expressions show that in the S-shell model all information about nuclear structure is contained in the form factor $R_{S}(Q)$. It divides out in the expression for the asymmetry (eq.(15)); thus, $A_{y}$ is given in terms of the free $\pi N$ scattering amplitudes only.

\section{RESULTS AND DISCUSSION}

\section{A. Pion scattering on unpolarized targets}

We begin our discussion with the analysis of some main features of the pion-nuclear interaction in the energy region of $T_{\pi}=100-300 \mathrm{MeV}$. The corresponding results of our calculations are depicted in Fig.1. 
One of the important properties of the $\pi N$ interaction in this energy region is the importance of the $\Delta$-isobar excitation, especially around $T_{\pi}=200 \mathrm{MeV}$ and, consequently, the large contribution from pion $p$-waves. This feature is reflected in the coherent scattering process which is proportional to $A$ (nuclear mass number) and described by the scalar part ( $\left.A_{00}\right)$ of the $\pi N$ amplitude. Since the $p$-wave part of this amplitude has a $\cos \Theta$ dependence ( see Appendix) the differential cross section experiences a minimum around $\Theta=90^{\circ}$. The position of this minimum is in fact shifted due to the Lorentz transformation of the pion angle from the $\pi N$ to the $\pi-{ }^{3} \mathrm{He}$ c.m. frame and due to the $s^{-}$, and $p$-waves interference. With increasing pion energy the minimum disappears because the contributions of the other multipoles become larger.

The spin-flip transitions due to the amplitudes $A_{10}$ and $A_{11}$ are proportional to $\sin \Theta$ which in the $\Delta$-resonance region fills in the minimum. Note that for $\pi^{-}$scattering the minimum is filled in more than for $\pi^{+}$scattering. This can easily be understood in the framework of the S-shell model where the spins of the two protons are coupled to zero. Therefore, due to the Pauli principle the spin-flip transition can be realized only through the neutron distribution in ${ }^{3} \mathrm{He}$. However, it is well known that in the $\Delta$-resonance region the $\pi^{-} n$ interaction is about 10 times stronger than the $\pi^{+} n$ one. Hence the strength of the spin-flip transition in $\pi^{-3}$ He scattering is about one order of magnitude larger than in $\pi^{+3}$ He scattering.

In Fig.1 we compare the results obtained with correlated three-body Fadeev wave functions with calculations performed in the simple S-shell model. At pion energies up to $T_{\pi}=300$ $\mathrm{MeV}$ the momentum transfer to the nucleus approaches $Q=3.6 \mathrm{fm}^{-1}$ which is not yet sufficient to differentiate between the phenomenological and the full three-body model. At $T_{\pi}=100 \mathrm{MeV}$ even the simple S-shell model agrees well with our full calculation. Thus, at this low energy plane wave calculations with S-shell harmonic oscillator wave functions are sufficient not only for a qualitative but also for a quantitative discussion of the $\pi^{+3} \mathrm{He}$ interaction. The agreement with experimental data from Refs. 41,42] in all three models is very good. 
Moving into the region of the $\Delta$-isobar excitation the contribution of pion rescattering becomes larger and reaches its maximum at $T_{\pi}=180-200 \mathrm{MeV}$. At backward angles the Sshell model calculations (with rescattering) deviate significantly from our full calculations in the three-body model indicating that S-shell harmonic oscillator wave functions have become insufficient to describe the nuclear wave function. At $T_{\pi}=295 \mathrm{MeV}$ the full calculations fail to reproduce the experimental data from Ref. [41] at large angles both in magnitude and in the shape of the differential cross section. The measurements indicate an additional dip around $\Theta=120^{\circ}$ while our calculations yield smooth predictions for $d \sigma / d \Omega$. Furthermore, around $\Theta=90^{\circ}$ our computation underestimates the data in $\pi^{-}$scattering but overestimates them in $\pi^{+}$scattering. Measurements of polarization observables may be useful in this kinematic region since asymmetries tend to be large where angular distributions have minima and could thus be sensitive to subtleties in the reaction mechanism.

We developed a similar approach for the pion-deuteron interaction using a two-body wave function generated by the Paris potential [43]. In Fig.2 we present our results for $\pi^{+} d$ elastic scattering at $T_{\pi}=180-300 \mathrm{MeV}$. Here the agreement with experimental data is excellent even at backward angles. Note that the main reason of that may be connected with the dominance of $\Delta$-resonance contribution. In this case as have been shown in Ref. [45] two-body approach is a good approximation for the more elaborate three-body Fadeev calculations.

However at lower energies $\left(T_{\pi}<50 \mathrm{MeV}\right)$ our model fails to reproduce the experimental data for the elastic $\pi d$ scattering. This is due to the well known problem of the description of the $S$-wave pion-nucleon interaction in nuclei with zero isospin. In such nuclei the contribution from the large isovector $\pi N$ scattering lengths are canceled. Therefore the role of higher order effects in the pion-nuclear potentials become important. But in the case of ${ }^{3} \mathrm{He}$ at low energies the scattering length is entirely formed by the isovector part of pion S-wave scattering on the additional proton. Therefore in this case our model with first order optical potential works much better [19].

Since $\pi d$ scattering in contrast to $\pi^{3} \mathrm{He}$ is realized only via isoscalar transitions we 
assume the discrepancy at backward angles in $\pi^{3} \mathrm{He}$ elastic scattering at $T_{\pi}=295 \mathrm{MeV}$ to be due to isovector second order effects. We will continue discussing this issue in subsection $\mathrm{C}$ where we consider the pion charge exchange reaction which is described solely by the isovector part of the pion-nuclear interaction.

Fig.3 presents our results for higher pion energies, $T_{\pi}=350-500 \mathrm{MeV}$; experiments in this energy domain have been proposed at LAMPF. At these energies the momentum transfer to

${ }^{3} \mathrm{He}$ for backward pion angles approaches $Q=5 \mathrm{fm}^{-1}$. These Q-values are large enough for differences between our three-body and phenomenological model to become visible. Therefore, applying phenomenological models in this region becomes questionable since at these momentum transfers meson exchange currents give important contributions to the charge form factors.

As mentioned before the $p$-wave dominance of the pion-nuclear interaction decreases with increasing of pion energy but it does not disappear entirely. There is still a noticeable deviation from the exponential fall of $d \sigma / d \Omega$ around $\Theta=90^{\circ}$ in terms of a broad bump. At larger angles the angular distributions obtained with both models go through a minimum that comes from the $\mathrm{C} 0$ nuclear form factor. However, even though the phenomenological model predicts this dip at smaller angles than the three-body model the $\pi^{+}$scattering data at $T_{\pi}=350 \mathrm{MeV}$ suggest a minimum at even smaller pion angles. No conclusions are possible for $\pi^{-}$scattering or higher pion energies since the experimental information is insufficient.

\section{B. Asymmetry and superratio.}

In Fig.4, we present our results for the asymmetry $A_{y}$ at $T_{\pi}=100,300$ and $500 \mathrm{MeV}$ both for $\pi^{+}$and $\pi^{-}$scattering. In $\pi^{+}$scattering $A_{y}$ approaches its maximum value of almost +1 at $T_{\pi}=100 \mathrm{MeV}$ around $\Theta=90^{\circ}$ (Fig. 4a). This is due again to the $p$-wave dominance of the $\pi N$ interaction which also causes the differential cross section to go through a minimum near $90^{\circ}$. This large asymmetry is not obvious since the analyzing powers of the elementary $\pi N$ reactions are quite small. In fact, $-0.2<A_{y}<0.08$ in $\pi^{+} n$ scattering and $0<A_{y}<0.5$ in 
$\pi^{+} p$ scattering. This indicates that the view of ${ }^{3} \mathrm{He}$ as a neutron target in the case of elastic pion scattering would lead to the wrong conclusions. While the spin-flip amplitude is similar to that of a neutron target, the non-spin-flip term is quite different. A detailed analysis of the asymmetry in this region has been performed in a previous paper [20]. Up to $T_{\pi}=180$ $\mathrm{MeV}$ the results of the simple S-shell model are very similar to the full calculations in the three-body model. Therefore, we conclude that in this region the asymmetry contains very little nuclear structure information. Furthermore, the pion rescattering effects are minimal at these energies.

However, this situation changes drastically at higher energies. In the full calculation $A_{y}$ goes through zero around $T_{\pi}=200 \mathrm{MeV}$ and becomes large and negative in the $260-300 \mathrm{MeV}$ region. In contrast, the asymmetry in the simple model remains positive at higher energies. As discussed in ref. [20] pion rescattering is mainly responsible for this effect.

Our results at $T_{\pi}=100 \mathrm{MeV}$ have been confirmed by first experimental measurements from TRIUMF [42]. As can be seen from Fig.4a, except for the fact that the maximum of the calculated asymmetry $A_{y}$ appears to lie at slightly smaller $\Theta$, the agreement with the data is very good.

In case of $\pi^{-}$scattering at $T_{\pi}=100 \mathrm{MeV}$ (Fig.4b) the difference between the simple model and the full calculation is larger than for the $\pi^{+}$case. This is caused mainly by the larger influence of pion rescattering. At $\Theta=90^{\circ}$ the differential cross section shows no minimum in contrast to $\pi^{+}$scattering. Therefore, the absolute value of $A_{y}$ is smaller and it has a less pronounced structure in the angular distribution.

In the high energy region around $T_{\pi}=500 \mathrm{MeV}$ the contribution of the $D_{13}(1525)$ resonance to the spin-flip part of the elementary amplitude becomes important and $A_{10}$ and $A_{11}$ can approximately be written as $A_{11}=-A_{10} \approx D_{13} \cos \Theta$ (see Appendix). Thus, for $\pi^{+}$scattering the spin-flip amplitude $G$ is proportional to $\left(A_{10}-A_{11}\right)$ while in case of $\pi^{-}$ scattering $G$ is proportional to $\left(A_{10}+A_{11}\right)$ which vanishes. Therefore, the asymmetry for $\pi^{-}$scattering at $T_{\pi}=500 \mathrm{MeV}$ is zero almost everywhere in the simple S-shell model. However, this exact cancellation is destroyed in the forward direction by pion rescattering and 
at backward angles by the D-state components of the ${ }^{3} \mathrm{He}$ wave function.

In the case of $\pi^{+}$scattering at $T_{\pi}=500 \mathrm{MeV}$, where $G \approx D_{13} \cos \Theta$, the asymmetry in the forward direction is large and it depends neither on the nuclear model nor on pion rescattering. Note that similar results for the asymmetry at $T_{\pi}=500 \mathrm{MeV}$ have been obtained by Chakravarti et al. [47]. Thus, in this kinematical region the asymmetry can be described directly via the elementary amplitude in accordance with eq.(27). Measurements in this region could extract information on the $D_{13}$ resonance in the nuclear medium.

Concluding our analysis of the elastic channel in the $\pi^{3} \mathrm{He}$ interaction we briefly consider the so-called "superratio"

$$
R=\frac{d \sigma\left(\pi^{+3} \mathrm{H}\right) d \sigma\left(\pi^{-3} \mathrm{H}\right)}{d \sigma\left(\pi^{+3} \mathrm{He}\right) d \sigma\left(\pi^{-3} \mathrm{He}\right)}
$$

discussed in detail in Ref. [11]. The main point of interest is related to the attempt to find charge symmetry breaking in the strong interaction. It is expected that $R$ is insensitive to the model uncertainties in the pion-nuclear interaction and to the Coulomb interaction. Therefore, in accordance with the relations (11) this ratio would have to be 1 at all angles and energies. But measurements 48 50 obtained a value $R>1$ along with angular and energy dependence. The generally accepted explanation [11,51] for this significant charge symmetry breaking effect assumes that the $2 \%$ difference in the ${ }^{3} \mathrm{He}$ and ${ }^{3} \mathrm{H}$ radius caused by the Coulomb repulsion between the two protons in ${ }^{3} \mathrm{He}$ is responsible. In Fig. 5 we compare our full model with the results of our calculations in the S-shell model using

1) $b=1.65 \mathrm{fm}$ both for ${ }^{3} \mathrm{H}$ and ${ }^{3} \mathrm{He}$ (dashed curves)

2) $b=1.65 \mathrm{fm}$ for ${ }^{3} \mathrm{H}$ and $b=1.68 \mathrm{fm}$ for ${ }^{3} \mathrm{He}$ (dash-dotted curves).

Using different harmonic oscillator parameters for ${ }^{3} \mathrm{H}$ and ${ }^{3} \mathrm{He}$ allows us to qualitatively explain the measured values for $R$, except for the region where the differential cross sections have the minimum. The deviation from experiment at larger angles is clearly due to the fact that harmonic oscillator wave functions at backward angles are inappropriate at these momentum transfers (which follows from our analysis of differential cross sections in the previous subsection). In this region we certainly require a realistic three-body wave function 
of ${ }^{3} \mathrm{He}$ that includes the additional Coulomb interaction between the two protons [52], like the one used by Gibbs and Gibson [11. Our three-body model gives $R \approx 1$ at all angles ( except small angles).

In Fig. 6 we show the differential cross sections at $T_{\pi}=180 \mathrm{MeV}$ for all of the 4 reactions entering the superratio calculated with isospin symmetric Fadeev wave functions. At backward angles preliminary data of Briscoe et al. [50] show deviation from our calculations which we did not find for the $T_{\pi}=200 \mathrm{MeV}$ data in Fig.1. As in our discussion about the discrepancies with the $T_{\pi}=295 \mathrm{MeV}$ data, we find in accordance to Ref. [14 the need of second order effects. To illustrate this we apply the $\rho^{2}$-term of the pion-nucleus interaction from Ref. [11]. In this way we obtain a better agreement with the data, however, in the case $\pi^{-3} \mathrm{H}$ and $\pi^{+3} \mathrm{He}$ a large deviation remains at $\Theta>150^{\circ}$.

\section{Pion single charge exchange}

In the previous subsections we have demonstrated that our formalism generally gives a good description of the elastic scattering data both for $\pi^{+}$and $\pi^{-}$at pion angles $0^{\circ}<\Theta<120^{\circ}$. Assuming charge symmetry it follows that the isovector part of the pionnuclear interaction which is responsible for the pion single charge exchange (SCE) reaction is accurately described in this kinematical region. Therefore we should not encounter serious difficulties in the description of the SCE process.

The results of our coupled channels calculations for ${ }^{3} \mathrm{H}\left(\pi^{+}, \pi^{0}\right)^{3} \mathrm{He}$ at $T_{\pi}=130 \mathrm{MeV}$ and for ${ }^{3} \mathrm{He}\left(\pi^{-}, \pi^{0}\right)^{3} \mathrm{H}$ at $T_{\pi}=200 \mathrm{MeV}$ are presented in Fig.7. Note that the rescattering contributions in SCE are larger than in the elastic channel. This is due mainly to the incoherent nature of the SCE process. Therefore, the influence of the elastic channel (proportional to $A$ ) on the pion rescattering term becomes enhanced. Experimental data at backward angles obtained in Ref. [53] by detecting the recoiling ${ }^{3} \mathrm{He}$ are in excellent agreement with our calculations performed in the framework of the three-body model. Similar results have been obtained by Landau [4.6] with phenomenological nuclear form factors. 
The SCE reaction on ${ }^{3} \mathrm{He}$ at $T_{\pi}=200 \mathrm{MeV}$ is at the present time the only example where experimental data at forward angles have become available [54 by directly detecting the $\pi^{0}$. The comparison with our full calculation shows a very good agreement up to $\Theta=60-70^{\circ}$. However, at larger angles there is a small deviation which, as we will see below, grows with increasing pion energy.

In Fig.7 we also show the contribution from the spin-flip and non-spin-flip part of the elementary $\pi N$ scattering amplitude separately. As in the case of elastic scattering the nonspin-flip transition experiences a minimum around $\Theta=90^{\circ}$ due again to the $p$-wave nature of the $\pi N$ interaction. The spin-flip contribution which has a $\sin \Theta$ angular dependence fills in this minimum. Note that for the SCE reaction the relative strength of the spin-flip transition is larger compared to elastic scattering. This can be attributed to the incoherent nature of both the spin-flip and the non-spin-flip mechanism of the SCE process.

Fig.7 demonstrates that there is interference between spin-flip and non-spin-flip transitions due entirely to rescattering effects. For example, the non-spin-flip transition in PWIA can be realized only through the non-spin-flip part of the $\pi N$ amplitude; in this case no interference is present. However, if pion rescattering is taken into account in a coupled channels framework the non-spin-flip transitions can be realized through double spin-flip transitions as well.

We now proceed to consider the high-energy region. The results depicted in Fig.8 show dramatic discrepancies between theory and experiment. Our coupled-channels calculations with three-body wave functions underestimate the data at backward angles about two orders of magnitude at $T_{\pi}=285 \mathrm{MeV}$ and about one order of magnitude at higher energies.

At present there is no explanation for this disagreement except some calculations in a Glauber multiple-scattering formalism performed in Refs. [12, 13]. However, we feel that this approach is inappropriate for an analysis in the large scattering-angle region.

As discussed in subsection $B$, one of the reasons for such a large discrepancy could be due to second-order contributions in the isovector part of the pion-nuclear interaction. A microscopic analysis of corresponding effects in $\pi^{3} \mathrm{He}$ scattering has been performed by 
Wakamatsu [14]. However, the contributions he found are not large enough to explain discrepancies of two orders of magnitude. One can remove this discrepancy by artificially enhancing the isovector second-order interaction whose influence on the SCE reactions is much stronger than on elastic ones. To check this assumption qualitatively we followed the prescription given in Refs. [8, 14] and introduced a second-order potential of the form

$$
\mathrm{V}_{\pi^{\prime} \pi}^{(2)}\left(\vec{q}^{\prime}, \vec{q}\right) \sim(A-1)^{2}\left(B_{1}+C_{1} \overrightarrow{q^{\prime}} \cdot \vec{q}\right) \exp \left(-\frac{b Q^{2}}{8}\right) \vec{\tau} \cdot \vec{t}
$$

with real parameters $B_{1}$ and $C_{1}$. Treating $B_{1}$ and $C_{1}$ as free parameters we extract $B_{1}=$ $0.086 / m_{\pi}^{4}$ and $C_{1}=-0.058 / m_{\pi}^{6}$ from a fit to the SCE data. These values are of similar magnitude as the parameters $B_{0}$ and $C_{0}$ extracted from an isoscalar second-order potential in Ref. [8]. In principle, $B_{1}$ and $C_{1}$ would have to be complex. However, a microscopic derivation of $V^{(2)}$ would in some way involve the square of the elementary $\pi N$ t-matrix and since the imaginary part dominates this amplitude around $T_{\pi}=300 \mathrm{MeV}$ the main contribution to $B_{1}$ and $C_{1}$ should be real. The results of our calculation including $V^{(2)}$ are shown in Fig.9. We confirm that the contribution of this potential in the charge-exchange channel is in fact much larger than in the elastic one. At the same time the second-order effects in elastic scattering at backward angles are of the same order as the discrepancy with experimental data. Thus, the longstanding problem in the description of the SCE reaction and elastic scattering at $T_{\pi}=300 \mathrm{MeV}$ at backward angles might have the same origin. Again, an isoscalar second-order potential [8] has to be included in the elastic scattering reactions before final conclusions can be drawn. To clarify the situation additional theoretical studies and experimental measurements in this region are required.

Note that the necessity of a second order potential follows also from three-body unitarity which requires to extend the nuclear model space by including the breakup channels. Our results show that the coupling with this channels is probably very important at large momentum transfer, in particular for SCE reactions at high energies and backward angles.

In Fig. 10 we present results for the asymmetry $A_{y}$ in the SCE reaction on ${ }^{3} \mathrm{He}$ at $T_{\pi}=100$, 300 and $500 \mathrm{MeV}$. As in the case of elastic scattering at $T_{\pi}=100 \mathrm{MeV}$ the simple S-shell 
model approximately reproduces the results of the full calculation. The small difference is caused mainly by pion rescattering. Note that if in eq.(28) for the simple model we neglect the small kinematic correction from the factor $W_{B}$ we obtain a simple relation between $A_{y}$ for ${ }^{3} \mathrm{He}$ and the elementary asymmetry:

$$
A_{y}\left({ }^{3} \mathrm{He}\right) \approx-A_{y}(p)
$$

The minus sign in this relation results from the opposite sign between spin-flip and nonspin-flip nuclear matrix elements (see eq.(26)) in ${ }^{3} \mathrm{He}\left(\pi^{-}, \pi^{0}\right)^{3} \mathrm{H}$ compared to the free process $p\left(\pi^{-}, \pi^{0}\right) n$.

Moving into the $\Delta$ resonance region the pion rescattering contribution becomes enhanced changing the sign of the asymmetry around $T_{\pi}=220 \mathrm{MeV}$. However, increasing the pion energy decreases the role of pion rescattering. Therefore, at $T_{\pi}=500 \mathrm{MeV}$ in the forward

direction the simple S-shell model results are again close to the full calculations. In this region the simple relation (31) is again fulfilled as in the case of $T_{\pi}=100 \mathrm{MeV}$. However, while at lower energies the role of the $P_{33}$ wave was dominating $A_{y}$, at $500 \mathrm{MeV}$ it is the $D_{13}$ resonance which has become important.

\section{Pion induced eta production}

Eta production by pions is another important $\pi N$ inelastic channel. Interest in the physics with eta mesons has grown significantly in recent years, experiments using hadronic probes to produce $\eta$ mesons have been performed at LAMPF, Brookhaven and Saclay. On the other hand, electron accelerators such as BATES, ELSA and the Mainz Microtron open the possibility to produce eta mesons with electrons or real photons.

At the present time little is known about the nature of the eta-nucleus interaction. For the elementary $\pi N \rightarrow \eta N$ process the experimental data is much less complete and accurate in contrast to $\pi N$-scattering data. There are also only few theoretical investigations of this reaction [22 24] based on the coupled channel isobar model for the $\pi N, \eta N$ and $\pi \pi N$ 
systems without background. In accordance with a recent analysis by Benmerrouche and Mukhopadhyay [57], where eta photoproduction has been studied, the role of background could be important. However, the corresponding contribution strongly depends on the value of the not well-defined $\eta N N$ coupling constant $g_{\eta}\left(0.6 \leq g_{\eta}^{2} / 4 \pi \leq 6.3\right)$. In such a situation the investigations of pion induced eta production on lightest nuclei could give additional information about the elementary amplitude. But before that we have to be sure that all other ingredients connected with the reaction mechanism and nuclear structure input are well under control.

Below we will concentrate mainly on the study of the initial and final state interaction and nuclear structure effects. The elementary processes with eta mesons will be described in the framework of the coupled channels isobar model of Ref. 224] with parameters extracted from available data ( see also Appendix).

The amplitudes for the nuclear processes have been obtained by solving the system of equations similar to eq.(10) but extended to include the $\eta$ channels. In this case ( omitting the index $L_{\pi}$ ) eq.(10) can be rewritten the following way

$$
F_{n^{\prime}, n}^{j, I}\left(q^{\prime}, q\right)=V_{n^{\prime}, n}^{j, I}\left(q^{\prime}, q\right)-\frac{a}{\pi} \sum_{n^{\prime \prime}} \int \frac{q^{\prime \prime 2}}{\mathcal{M}\left(q^{\prime \prime}\right)} \frac{V_{n^{\prime}, n^{\prime \prime}}^{j, I}\left(q^{\prime}, q^{\prime \prime}\right) F_{n^{\prime \prime}, n}^{j, I}\left(q^{\prime \prime}, q\right)}{E(q)-E\left(q^{\prime \prime}\right)+i \epsilon} d q^{\prime \prime}
$$

where $n=\pi, \eta$ with $\pi$ and $\eta$ labelling the $\pi A$ and $\eta A$ channels, respectively.

Since the isospin of the eta is zero, only contributions to the channel with total isospin $1 / 2$ are possible. The definition of the corresponding isospin dependent amplitudes $\mathrm{F}_{I}$ or $\mathrm{V}_{I}$ is the same as in eqs.(8) and (20). However,the isospin factors $C_{I}^{T}$ in eq.(20) have to be changed to

$$
C_{I}^{T}=\frac{1}{\sqrt{2}} \delta_{I, \frac{1}{2}} \delta_{T, 0}
$$

for $(\eta, \eta)$ scattering and for the $(\pi, \eta)$ reaction to

$$
C_{I}^{T}=-\frac{1}{\sqrt{2}} \delta_{I, \frac{1}{2}} \delta_{T, 1}
$$

Results of our calculations for the differential cross section at $T_{\pi}=555 \mathrm{MeV}$ are shown in Fig.11. First we point out that we achieve a good description of recent measurements 25] in 
the forward direction. Our agreement with the data is in contrast to the DWIA calculations in Ref. 25] which underestimate the data by a factor of about 3. The corrections from two-step processes, such as $\left(\pi^{-}, \pi^{0}\right)\left(\pi^{0}, \eta\right)$, are not very large (about $10 \%$ ). The main effect of the pion- and eta-nuclear interaction in the initial and final state is to fill in the diffraction minimum in the differential cross section.

The situation changes dramatically in the backward direction. Here our full calculation with three-body wave functions underestimates the experimental measurements by a factor of about 50. Employing phenomenological nuclear form factors for the $\mathrm{J}=0$ transition densities reduces the disagreement with the data to a factor of $2-3$, similar to the findings of Ref. 25]. However, the application of the phenomenological approach, as mentioned before, is questionable because we are again in a high momentum transfer region. The origin of this discrepancy may be similar to the one encountered in the pion SCE reaction.

In contrast to the differential cross section the asymmetry $A_{y}$ is less sensitive to the details of nuclear structure. Therefore, in the forward direction, as in the pion SCE reaction, we can approximately write

$$
A_{y}^{\pi, \eta}\left({ }^{3} \mathrm{He}\right) \approx-A_{y}^{\pi, \eta}(p)
$$

Fig.12 presents the dependence of the differential cross section at $\Theta_{\eta}=0^{\circ}$ on the eta momentum in the $\eta^{3}$ He c.m. system. These data can be reproduced only for $q_{\eta}>150$ $\mathrm{MeV} / \mathrm{c}$ which corresponds to $T_{\pi}>490 \mathrm{MeV}$, while at lower eta momenta our calculations (similar to Ref. [25]) significantly underestimate the observed cross section regardless which nuclear model is used.

As pointed out in Ref. 25] this is a region where the $(\pi, \eta)$ reaction proceeds below the free eta production threshold. Here the corrections to the impulse approximation as well as different off-shell behaviors of the elementary amplitude could be important. These effects may lead to significant enhancements of the cross section similar to those observed, for example, in $\pi^{0}$ - photoproduction at threshold on very light nuclei. [58] 


\section{CONCLUSION}

In this paper we have studied the interaction of pions with ${ }^{3} \mathrm{He}$ and ${ }^{3} \mathrm{H}$ in a coupled channels, multiple-scattering approach carried out in momentum space. Our investigation covered a wide energy region; from $T_{\pi}=100 \mathrm{MeV}$ - well below the $\Delta$ resonance - beyond the $\Delta$ region into the domain of the $D_{13}(1525)$ resonance and up to the $\eta$ production threshold. Correlated three-body Fadeev wave functions were employed to describe the trinucleon ground state. Phase-shift parameterizations were used for the elementary $\pi N$ amplitudes, along with a separable potential for the off-shell extrapolation. The eta-production channel, $\pi N \rightarrow \eta N$, was described in an isobar model that includes the $S_{11}(1535), P_{11}(1440)$ and $D_{13}(1525)$ resonances as $\mathrm{S}-, \mathrm{P}-$, and $\mathrm{D}$-wave interactions and reproduces available low energy $\pi^{-} p \rightarrow \eta n$ cross section data.

The $\pi^{+}$and $\pi^{-}$elastic scattering on ${ }^{3} \mathrm{He}$ were well reproduced in our model in almost the entire kinematical region considered here. Only for pion kinetic energies above $180 \mathrm{MeV}$ and backward pion angles - a region with few experimental data - hints of an inadequate description appear. In this kinematical region phenomenological $C 0$ nuclear form factors extracted from charge form factors should not be used any more because MEC contributions have become substantial. The asymmetries for $\pi^{+3}$ He elastic scattering are large in contrast to asymmetries measured on p-shell nuclei. Noteworthy is the change of sign in $A_{y}$ from +1 to -1 when one moves into the $\Delta$-resonance region; this effect - caused by pion multiple scattering - should be verified experimentally. At $T_{\pi}=500 \mathrm{MeV}$ and in the forward direction the asymmetry is entirely determined by the $D_{13}$ resonance contribution.

In contrast to pion elastic scattering the pion single charge exchange calculations agree with the data only up to $T_{\pi}=200 \mathrm{MeV}$; at higher energy the theoretical description dramatically fails to explain the measurements by underestimating them up to two orders of magnitude. Since the $\pi N$ amplitudes and the nuclear wave function is presumably well known this discrepancy may be an indication for two- and three-body processes that go beyond the impulse approximation. We note that a similar phenomenon has been observed 
in the photoproduction process ${ }^{3} \mathrm{He}\left(\gamma, \pi^{+}\right)^{3} \mathrm{H}$ at large momentum transfers. Since, on the other hand, $\pi^{+} d$ elastic scattering data can be reproduced very well in our model we have introduced a phenomenological isovector second-order potential and adjusted the parameters to reproduce the SCE data. This is very much connected to the problems we have found for back-angle elastic scattering at $T_{\pi}=180 \mathrm{MeV}$. Further investigations of second-order potential has to be done. Clearly, it would be desirable to derive such a potential microscopically. Again, the asymmetry of the process ${ }^{3} \mathrm{He}\left(\pi^{-}, \pi^{0}\right)^{3} \mathrm{H}$ is predicted to be large.

Finally, we discussed pion induced eta production, ${ }^{3} \mathrm{He}\left(\pi^{-}, \eta\right)^{3} \mathrm{H}$, in the framework of our coupled-channels model. At $T_{\pi}<490 \mathrm{MeV}$ which is a region below the free production threshold our results for forward eta production significantly underestimate the data. We found good agreement with the few available data at small momentum transfer and $T_{\pi}>560$ $\mathrm{MeV}$ but large deviations in the backward direction with large $\mathrm{Q}$. We believe that the same mechanism in both incoherent processes is responsible for this puzzle. Future theoretical studies should reveal if these discrepancies present a clear indication of a breakdown in the impulse approximation. Very recently a paper by L. C. Liu has been published which confirms our conclusion on the importance of two-nucleon effects in nuclear eta production [59].

\section{ACKNOWLEDGMENTS}

We want to thank W. J. Briscoe and S. K. Matthews for discussions and for providing us with their preliminary data and R. Mach for discussions on the pion-nuclear interaction.

This work was supported by the Deutsche Forschunsgemeinschaft (SFB201) and the Natural Science and Engineering Research Council of Canada (NSERC).

In this appendix we define our partial wave amplitudes for $\pi N$ scattering, eta production and $\eta N$ scattering which in the past have been given by different authors using various 
conventions.

As in eq.(4) the elementary amplitudes for all three processes are defined as:

$$
\hat{f}_{m, n}=A_{00}+A_{01} \vec{t}_{m, n} \cdot \vec{\tau}+i \vec{\sigma} \cdot\left[\hat{\vec{q}}_{i} \times \hat{\vec{q}}_{f}\right]\left(A_{10}+A_{11} \vec{t}_{m, n} \cdot \vec{\tau}\right)
$$

where $n$ and $m$ label the $\pi$ or $\eta$ mesons. Here we introduce the auxiliary matrix $\vec{t}_{m, n}$. The cyclic components of this matrix are:

1)for $\pi N$ scattering $\left(\vec{t}_{\pi, \pi}\right)_{\lambda}=(\vec{t})_{\lambda}$ is the standard pion isospin operator;

2) for the $\pi N \leftrightarrow \eta N$ reaction $\left(\vec{t}_{\pi, \eta}\right)_{\lambda}=(-1)^{\lambda}$ and

3) for $\eta N$-scattering $\left(\vec{t}_{\eta, \eta}\right)_{\lambda}=0$.

The differential cross section for the elementary processes in the meson-nucleon c.m. system is given as

$$
\frac{d \sigma_{m, n}}{d \Omega}=\frac{q_{f}}{2 q_{i}} \operatorname{Tr}\left[f_{m, n}^{+} f_{m, n}\right]
$$

The expansion of the $A_{S T}$ amplitudes in partial amplitudes with orbital angular momentum $l$ and total angular momentum $j=l \pm \frac{1}{2}$ is identical for all three processes: for non spin-flip $(S=0)$ amplitudes

$$
A_{0 T}=\sum_{l}\left[(l+1) f_{l T}^{(+)}(w)+l f_{l T}^{(-)}(w)\right] P_{l}(\cos \theta)
$$

and for spin-flip amplitudes

$$
A_{1 T}=\sum_{l}\left[f_{l T}^{(+)}(w)-f_{l T}^{(-)}(w)\right] P_{l}^{\prime}(\cos \theta)
$$

The main difference arising in the formalism for $\pi N$ scattering, $\eta N$ scattering and $\pi N \leftrightarrow$ $\eta N$ lies in the expansion of the partial amplitudes $f_{l T}^{( \pm)}$in terms of contributions with total isospin $I=\frac{1}{2}\left(f_{l \pm}^{1 / 2}\right)$ and $I=\frac{3}{2}\left(f_{l \pm}^{3 / 2}\right)$; where \pm corresponds to $j=l \pm \frac{1}{2}$. Therefore, below these reactions are presented separately.

\section{1. $\pi N$ scattering}

$$
f_{l 0}^{( \pm)}=\frac{1}{3}\left(2 f_{l \pm}^{3 / 2}+f_{l \pm}^{1 / 2}\right) \quad \text { and } \quad f_{l 1}^{( \pm)}=\frac{1}{3}\left(f_{l \pm}^{3 / 2}-f_{l \pm}^{1 / 2}\right)
$$


Keeping only the contributions from $S, P$ and $D$ waves in the expansions (A3-A4) and using standard notation, $f_{l j}^{I} \equiv l_{2 I, 2 j}$, for the $A_{S T}$ amplitudes we arrive at

$$
\begin{aligned}
3 A_{00}= & 2 S_{31}+S_{11}+\left(4 P_{33}+2 P_{13}+2 P_{31}+P_{11}\right) \cos \theta+ \\
& \left(3 D_{15}+2 D_{13}\right) P_{2}(\cos \theta) \\
3 A_{01}= & S_{31}-S_{11}+\left(2 P_{33}-2 P_{13}+P_{31}-P_{11}\right) \cos \theta- \\
& \left(3 D_{15}+2 D_{13}\right) P_{2}(\cos \theta) \\
3 A_{10}= & 2 P_{33}+P_{13}-2 P_{31}-P_{11}+3\left(D_{15}-D_{13}\right) \cos \theta \\
3 A_{11}= & P_{33}-P_{13}-P_{31}+P_{11}-3\left(D_{15}-D_{13}\right) \cos \theta .
\end{aligned}
$$

\section{2. $\pi N \leftrightarrow \eta N$ reaction}

In this case due to the isospin zero nature of the eta meson only total isospin $I=\frac{1}{2}$ is allowed. Therefore, in accordance with definition (8) we obtain:

$$
f_{l 0}^{( \pm)}=0 \quad \text { and } \quad f_{l 1}^{( \pm)}=-\frac{1}{\sqrt{3}} f_{l \pm}^{1 / 2}
$$

The amplitude $\hat{f}_{\pi, \eta}$ in this process is purely isovector, e.g. $A_{00}^{\pi, \eta}=A_{10}^{\pi, \eta}=0$. The isovector amplitudes $A_{01}^{\pi, \eta}$ and $A_{11}^{\pi, \eta}$ are given by

$$
\begin{gathered}
\sqrt{3} A_{01}^{\pi, \eta}=-\left(S_{11}^{\pi, \eta}+P_{11}^{\pi, \eta} \cos \theta+2 D_{13}^{\pi, \eta} P_{2}(\cos \theta)\right) \\
\sqrt{3} A_{11}^{\pi, \eta}=P_{11}^{\pi, \eta}+3 D_{13}^{\pi, \eta} \cos \theta
\end{gathered}
$$

where we have neglected $P_{13}$ and $D_{15}$ contributions.

\section{3. $\eta N$ scattering}


In this case the amplitude $\hat{f}_{\eta, \eta}$ consists of an isoscalar part only. Therefore,

$$
f_{l 0}^{( \pm)}=f_{l \pm}^{1 / 2} \quad \text { and } \quad f_{l 1}^{( \pm)}=0
$$

and

$$
\begin{gathered}
A_{00}^{\eta, \eta}=S_{11}^{\eta, \eta}+P_{11}^{\eta, \eta} \cos \theta+2 D_{13}^{\eta, \eta} P_{2}(\cos \theta) \\
A_{10}^{\eta, \eta}=-\left(P_{11}^{\eta, \eta}+3 D_{13}^{\eta, \eta} \cos \theta\right) .
\end{gathered}
$$

The expressions for the $S_{11}, P_{11}$ and $D_{13}$ amplitudes for $\pi N \leftrightarrow \eta N$ reaction and $\eta N$ scattering are taken from Ref. [24]. 


\section{REFERENCES}

* Permanent address is Laboratory of Theoretical Physics, JINR Dubna, Head Post Office Box 79, SU-101000 Moscow, Russia.

[1] T. Ericson and W. Weise, Pions and Nuclei (Clarendon press, Oxford,1988)

[2] A. K. Kerman, H. McManus, and R. M. Thaler, Ann. Phys. (N.Y.) 8, 551 (1955)

[3] M. L. Goldberger and K. M. Watson, Collision theory (Wiley, New York, 1964)

[4] R. H. Landau, Ann. Phys. (N.Y.) 92, 205 (1975)

[5] R. Mach, Nucl. Phys. A258, 513 (1976)

[6] R. H. Landau and A. W.Thomas, Nucl. Phys. A302, 461 (1978)

[7] L. C. Liu and C. M. Shakin, Prog. Part. Nucl. Phys. 5, 207 (1981)

[8] M. Gmitro, S. S. Kamalov, and R. Mach, Phys. Rev. C 36, 1105 (1987)

[9] Yong Rae Kwon and F. Tabakin, Phys. Rev. C 18, 932 (1978)

[10] A. Cieply, M. Gmitro, R. Mach, and S.S. Kamalov, Phys. Rev. C 44, 713 (1991)

[11] W. R. Gibbs and B. F. Gibson, Phys. Rev. C 43, 1012 (1991) and references contained therein

[12] K. Lohs and V. Mandelzweig, Z. Phys. A 283, 51 (1977)

[13] W. J. Gerace, J. P. Mestre, J. M. Walker, and D. A. Sparrow, Phys. Rev. C 22, 1197 (1980)

[14] M. Wakamatsu, Nucl. Phys. A 312, 427 (1978); 340, 289 (1980)

[15] T.-S. H. Lee and S. Chakravarti, Phys. Rev. C 16, 273 (1977)

[16] R. Mach and M. G. Sapozhnikov, J. Phys.G: Nucl. Phys. 10, 147 (1984)

[17] M. Kh. Khankhasayev, J. Adam, Jr., and E. Truhlik, Czech. J. Phys. B 35, 420 (1985) 
[18] W. R. Gibbs, B. F. Gibson, A. T. Hees, G. J. Stephenson, Jr., W. B. Kaufmann, Phys. Rev. C 13, 2433 (1976)

[19] S. S. Kamalov, L. Tiator, and C. Bennhold, Few-Body Systems 10, 143 (1991)

[20] C. Bennhold, B. K. Jennings, L. Tiator, and S. S. Kamalov, Nucl. Phys. A 540, 621 (1992)

[21] R. A. Brandenburg, Y. E. Kim, and A. Tubis, Phys. Rev. C 12, 1368 (1975)

[22] R. S. Bhalerao and L. C. Liu, Phys. Rev. Lett. 54, 865 (1985)

[23] H. C. Chang, E. Oset, and L. C. Liu, Phys. Rev. C 44, 738 (1991)

[24] C. Bennhold and Tanabe, Nucl. Phys. A 530, 625 (1991)

[25] C. Peng et al., Phys. Rev. Lett. 58, 2027 (1987); 63, 2353 (1989)

[26] M. Gmitro, J. Kvasil, and R. Mach, Phys. Rev. C 31, 1349 (1985)

[27] G. Rowe, M. Salamon, and R. H. Landau, Phys. Rev. C 18, 584 (1978)

[28] D. J. Herndon et al., LBL report UCRL-20030 $\pi N$

[29] Review of Particle Properties, Phys. Rev. D 45, VIII.10 (1992)

[30] R. A. Arndt, J. M. Ford, and L. D. Roper, Phys. Rev. D 32, 1085 (1985)

[31] R. A. Arndt, Z. Li, L. D. Roper, and R. L. Workman, Phys. Rev. D 43, 2131 (1991)

[32] J. T. Londergan, K. M. McVoy, and E. J. Moniz, Ann. Phys. (N.Y.) 78, 299 (1973)

[33] S. A. Gurvitz, Phys. Rev. C 33, 422 (1986)

[34] C. M. Vincent and S. C. Phatak, Phys. Rev. C 10, 391 (1974)

[35] L. Tiator, A. K. Rej, and D. Drechsel, Nucl. Phys. A333, 343 (1980)

[36] R. A. Eramzhyan, M. Gmitro, and S. S. Kamalov, Phys. Rev. C 41, 2865 (1991) 
[37] I. V. Falomkin et al., Nuovo Cimento, 57, 111 (1980)

[38] H. Henning, P. U. Sauer and W. Thies, Nucl. Phys. A 537, 367 (1992)

[39] J. S. McCarthy, I. Sick, and R. R. Whitney, Phys. Rev. C 15, 1396 (1977)

[40] F.-P. Juster et al., Phys. Rev. Lett. 55, 2261 (1985)

[41] J. Källne, J. F. Davis, J. S. McCarthy, R. C. Minehart, R. R. Whitney, R. L. Boudrie, J. B. McClelland, and A. Stetz, Phys. Lett. B 103, 13 (1981)

[42] B. Larson et al., Phys. Rev. Lett. 67, 3356 (1991)

[43] M. Lacombe, B. Loisean, R. VinhMau, J. Cote, P. Pires, and R. de Tourreil, Phys. Lett. B 101, $139(1981)$

[44] K. Gabathuler, J. Domingo, P. Gram, W. Hirt, G. Jones, P. Schwaller, J. Zichy, J. Bolger, O. Ingram, J. P. Albanese, and J. Arvieux, Nucl. Phys. A 350, 253 (1980)

[45] T.I. Kopaleishvili, Sov.J. Part. Nucl. 10 (2), 167 (1979)

[46] J. Boswell, G. S. Das, P. C. Gugelot, J. Källne, J. McCarthy, R. R. Whitney, J. E. Bolger, C. F. Moore, and C. A. Goulding, Nucl. Phys. A466, 458 (1987)

[47] S. Chakravarti, C.M. Edwards, D. Dehnhard, and M.A. Franey, Few-Body Systems, Suppl. 5, 267 (1992)

[48] C. Pillai, D. B. Barlow, B. L. Berman, W. J. Briscoe, A. Mokhtari, B. M. K. Nefkens, A. M. Petrov, and M. E. Sadler, Phys. Lett. B 207, 389 (1988)

[49] B. M. K. Nefkens, W. J. Briscoe, A. D. Eichon, D. H. Fitzgerald, A. Mokhtari, J. A. Wightman, and M. E. Sadler, Phys. C 41, 2770 (1990)

[50] W. J. Briscoe et al., Procceding of the International Nuclear Physics Conference, Wiesbaden, Germany, July 26 - August 1, 1992, to be published in Nucl. Phys A

[51] Y. E. Kim, M. Krell, and L. Tiator, Phys. Lett. B 172, 287 (1986) 
[52] J. L. Friar, B. F. Gibson, and G. L. Payne, Phys. Rev. C 35, 1502 (1987)

[53] P. Glodis et al., Phys. Rev. Lett. 44, 234 (1980)

[54] J. D. Bowman, Nucl. Phys. A335, 375 (1980)

[55] J. Källne, H. A. Thiessen, C. L. Morris, S. L. Verbeck, M. J. Devereaux, G. R. Burleson, J. S. McCarthy, R. R. Whitney, J. E. Bolger, C. F. Moore, and C. A. Goulding, Phys. Rev. Lett. 42, 159 (1979)

[56] L. Orphnos, J. C. McCarthy, R. C. Minehart, P. A. M. Gram, B. Höistad, C. F. Perdrisat, and J. Källne, Phys. Rev. C 26, 2111 (1982)

[57] M. Benmerrouche and N. C. Mukhopadhyay, Phys. Rev. Lett. 67, 1070 (1991)

[58] P. Argan et al., Phys. Rev. C 21, 1416 (1980)

[59] L. C. Liu, Phys. Lett. B 288, 18 (1992) 


\section{FIGURE CAPTIONS}

1. Differential cross sections for $\pi^{+}(\mathbf{a})$ and $\pi^{-}$(b) elastic scattering on ${ }^{3} \mathrm{He}$ at pion kinetic energies $T_{\pi}=100,200$ and $295 \mathrm{MeV}$ calculated with three-body (solid curves) and harmonic oscillator S-shell (dashed curves) wave functions. The dotted curves are the PWIA results obtained in the S-shell model. Experimental data are from Ref. [41] $(\bullet)$ and Ref. 42$]$ (o).

2. Differential cross sections for $\pi^{+}$elastic scattering on the deuteron at pion kinetic energies $T_{\pi}=180-300 \mathrm{MeV}$ calculated with Paris potential wave function [43]. Solid and dashed curves are full and PWIA calculations, respectively. Experimental data are from Ref. 444.

3. Same as in Fig. 1 for pion energies $T_{\pi}=350,400$, and $500 \mathrm{MeV}$. The dash-dotted curves are the results of calculations in the phenomenological model with $\mathrm{C} 0$ form factors extracted from charge distributions of ${ }^{3} \mathrm{He}$ and ${ }^{3} \mathrm{H}$. Experimental data are from Ref. [45].

4. The target asymmetry $A_{y}$ in $\pi^{+}(\mathbf{a})$ and $\pi^{-}$(b) elastic scattering on ${ }^{3} \mathrm{He}$ at $T_{\pi}=100$, 295 and $500 \mathrm{MeV}$. The notations for the curves are the same as in Fig.1. Experimental data at $T_{\pi}=100 \mathrm{MeV}$ are from Ref. [42].

5. Nuclear structure effects in the superratio. Solid curves are the results obtained using three-body wave functions and dashed curve - using S-shell wave functions with harmonic oscillator parameter $b=1.65 \mathrm{fm}$ both for the ${ }^{3} \mathrm{He}$ and ${ }^{3} \mathrm{H}$. Dash-dotted

curves are the S-shell model results with $b=1.65 \mathrm{fm}$ for ${ }^{3} \mathrm{H}$ and $b=1.68 \mathrm{fm}$ for ${ }^{3} \mathrm{He}$. Experimental data are Refs. [48] $(\times)$, [49](•) and [50](o).

6. Differential cross sections for $\pi^{ \pm}$elastic scattering on ${ }^{3} \mathrm{He}(\mathbf{a})$ and ${ }^{3} \mathrm{H}(\mathbf{b})$ at $T_{\pi}=180$ $\mathrm{MeV}$. The dashed and solid curves show our calculations using three-body wave functions with and without second-order potential from Ref. [8] respectively. Experimental data are from Ref. [49](•) and Ref. [50](o) (preliminary). 
7. Differential cross sections for ${ }^{3} \mathrm{H}\left(\pi^{+}, \pi^{0}\right)^{3} \mathrm{He}$ at $T_{\pi}=130 \mathrm{MeV}$ and ${ }^{3} \mathrm{He}\left(\pi^{-}, \pi^{0}\right)^{3} \mathrm{H}$ reaction at $T_{\pi}=200 \mathrm{MeV}$. In the latter case the contributions of spin-flip (long-dash-dotted) and non-spin-flip (long-dashed curve ) parts of $\pi N$ amplitude are shown separately. The notations of other curves are the same as in Fig.1. Experimental data for the $\left(\pi^{+}, \pi^{0}\right)$ reaction are from Ref. [53], data for the $\left(\pi^{-}, \pi^{0}\right)$ reaction are from Ref. [54](•) and Ref. 55 (o).

8. Differential cross sections for ${ }^{3} \mathrm{He}\left(\pi^{-}, \pi^{0}\right)^{3} \mathrm{H}$ at $T_{\pi}=285,428$ and $525 \mathrm{MeV}$ calculated in the three-body (solid curves ) and phenomenological (dash-dotted curves ) models. Experimental data are from Ref. [56].

9. Phenomenological isovector second-order contribution in SCE and elastic channels at $T_{\pi}=290 \mathrm{MeV}$. Solid and dashed curves are the results without and with $V^{(2)}$-term respectively. Experimental data are from [53] for SCE and 41] for elastic scattering.

10. The target asymmetry $A_{y}$ for ${ }^{3} \mathrm{He}\left(\pi^{-}, \pi^{0}\right)^{3} \mathrm{H}$ at $T_{\pi}=100,295$ and $500 \mathrm{MeV}$. The notations are the same as in Fig.1. The dash-dotted curves are the results of calculations in the phenomenological model.

11. Differential cross section (a) and target asymmetry (b) for ${ }^{3} \mathrm{He}\left(\pi^{-}, \eta\right)^{3} \mathrm{H}$ at $T_{\pi}=555$ MeV. The solid (dashed) curves are the results of full (PWIA) calculations with three-body wave functions and the dash-dotted curves are results obtained in the phenomenological model. The dotted curves are PWIA results obtained with S-shell wave functions. Experimental data are from Ref. [25].

12. Eta c.m. momentum dependence for the differential cross section of ${ }^{3} \mathrm{He}\left(\pi^{-}, \eta\right)^{3} \mathrm{H}$ at $0^{\circ}$. The dash-dotted and full curves show our full calculations using phenomenological and three-body wave functions, respectively. The dotted curve is obtained with three-body wave functions in PWIA. Experimental data are from Ref. [25]. 